\title{
Workplace Violence Faced by Nurses in a Rural Tertiary Hospital of Central India: Pattern \& Intervention
}

\author{
Anagha Abhoy Sinha* \\ Consultant Psychiatrist, India \\ *Corresponding author: Anagha Abhoy Sinha, Consultant Psychiatrist, Sinha Hospital, HTFT Complex, Mount Road, Sadar Nagpur, India
}

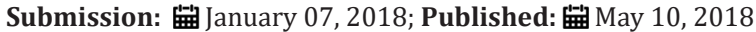

\section{Introduction}

It is often said about the nurses that they are strong enough to tolerate everything and soft enough to understand everyone. It would not be an overstatement to say that, in the hierarchy of the healthcare system, they are the most taken for granted population and thus one of the most vulnerable. In most countries, the nursing staffs have inflexible working hours; have maximum periods of interaction with the consumers of healthcare and are given a status of secondary citizens in comparison with their doctor counterparts. The power to exercise freedom of expression and peaceful assembly without apprehensions of violence or intimidation is regarded as a basic human right, by United Nations' universal declaration on human rights [1]. Workplace violence can be regarded as any act of aggression manifesting into a physical or emotional assault, towards a person on duty [2]. A work place can be regarded as any setting where a person renders his professional duties, which in the cases of nurses is mostly the hospital settings. It is inclusive of the entire work environment, such as the parking spaces or premises or even a temporary place of deputation for work purposes. The perpetrator may be any person who is the recipient of medical help or a senior or junior fellow worker, a member of the organization of work or even a random individual with no legitimate workplace relationship to the victim but merely a visitor in the hospital.

When we talk about violence, the first thing that strikes our minds is a brutal physical or at times sexual assault. Physical assault may result in serious bodily harm incurred as a result of biting, kicking, slapping, punching or throwing objects in the vicinity, on the victim. At times, it may even result in grievous disability or mortality. But there are several negativistic behaviours and acts that qualify as workplace violence. It could be in the form of verbal abuses and insults, shouting, sexually charged comments, intimidation or other threatening behaviour such as aggressive gesturing or stalking the worker, slamming doors or fisting. Behaviours from co-workers such as disobedience, discourteous behaviours, malicious remarks, maligning one's conduct, favouring some workers for work related perks and promotions. Any of these aggressive behaviours can prove to be detrimental to the psyche and professional performance of a worker. Overall, this results in decreased productivity, inefficiency and increased absenteeism to workplace. This affects the health care sector more than any other field as nurse is the backbone of the medical services, not to forget the personal losses incurred by the victim nurses [3]. Though not a new entity, workplace violence against nurses and other health workers has certainly been on the rise and is now considered a serious occupational hazard throughout the world [4]. Humiliation and behaviours causing loss of dignity to an individual can be forms of Psychological or Emotional violence. Discrimination or degradation of the workers in the work environment on the basis of ethnicity, colour or religious beliefs, can be categorized as racial violence.

By increasing awareness about these aggressive behaviours, nurses can be equipped to anticipate risk factors for an impending act of violence from patients, their relatives, subordinates and seniors $[5,6]$. Hence, we decided to take up a study to assess the pattern of violence faced by the Nurses in our setup. We would like to assess the data \& see what the most common form of violence used is \& how we could minimise it so that the healthcare professionals may be able to dispense best healthcare services to its service users. Finally we would like to assess as to how we can minimise all such violence \& make the work place environment safer [7].

\section{Aim and Objectives}

A. To examine the problem of violence in health care and its patterns, faced by nurses in their work setting.

B. To recognise the possible causative triggers.

C. To evaluate the probable ways to diminish the occurrence of this health hazard. 


\section{Methodology}

A. Study site: Acharya Vinoba Bhave Rural Hospital \& Jawaharlal Nehru Medical College

B. Type of study: Cross sectional

C. Subjects: Nurses involved inpatient care from various departments.

D. Type of sampling: Purposive

E. Total number of subjects: 60

F. Duration of study: 2 months (Jan-Feb 2016)

G. Instrument used: Workplace Violence in Health Sector country case study - Questionnaire.

\section{Inclusion Criteria}

A. Subjects consenting to be a part of the study \& willing to provide unbiased view via the answers in each section of questionnaire.

B. Subjects with a minimum of 1 years of experience in the hospital setup.

\section{Exclusion Criteria}

A. Subjects with less than 1 years of experience in the setup.

B. Subjects not willing to be a part of study.

\section{Learning Objectives}

From the above study, we will be able conclude the following

A. Prevalence of aggression against nursing staff in our hospital setup.

B. Common types of aggression encountered on day to day basis.

C. Most violent forms of aggression encountered.

D. Reason for such violence at the setup.

E. Strategies that need to be adopted to address all such issues and minimise such occurrences.

All such studies can help identify the reason for health care violence in the developing countries \& come to a consensus as to how the management protocol should be standardised for a better relationship with the patients and their families along with improvement of communication skills so as to reduce all such unpleasant happenings in hospitals \& most importantly, make the health care atmosphere pleasant for our Nurse to render selfless service to the patients.

\section{Tools Used}

The Workplace Violence in Health Sector country case studyQuestionnaire, used by The International Labour Office (ILO), International Council of Nurses (ICN), World Health Organization (WHO) and Public Services International (PSI), for their programme to minimize violence in health sector, was used in this study. The questionnaire consists of an initial section on the socio demographic profile of the participants. It also covers details of patient's working environment. The subsequent sections have questions pertaining to the type and pattern of violence incurred by the participant. Details regarding attacks which could be physical, psychological, verbal, sexual or intimidating acts like bullying or mobbing. A part of the questions help assess reporting or non reporting he incidents along with impact and measures taken for the same. The last section of the questionnaire asks relevant details about the participant's perception about the incident and suggestions about what could be done to minimise such acts of violence and aggression. SPSS Statistical Package for social sciences was used for statistical analyses.

\section{Ethical Implications}

All participants were required to give full, informed consent before taking part in the study. They were informed of their right to withdraw consent at anytime during the two month period, at which point their data was removed from the study. They were assured of confidentiality and anonymity.

\section{Interview Technique}

The socio demographic profiles of participants were recorded using a predesigned semi structured data sheet. The participating nurses were representative of surgical and non surgical departments. They were assigned the task of questionnaire completion during their working hours in the out - patient department or wards, keeping their convenience as a priority. They were requested to fill the questionnaires completely which were later submitted to our team of doctors.

\section{Discussion}

The common factors noted to be contributing towards violence against the nurses are:

Individual characteristics - substance use, organic and metabolic causes causing mental derangements, inexperience of staff, negligence.

Environment Characteristics- Stressful environment, shortage of staff

Relational Characteristics - poor rapport, short temper, low frustration tolerance.

Societal causes - ignorance, acceptance of violence, poor backing and hostility.

Several studies have been conducted worldwide, to assess the concern of violence against medical health professionals. Most of these studies concentrate on violence directed towards the health provider by the patient. There is paucity of data in the developing countries regarding this issue. The statistics do not represent a clear picture of the situation as most of the cases of violence against nurses go unreported and unrecognized. 
A study conducted in two public hospitals of the capital city of Riyadh in Saudi Arabia, reported that nurses were more predisposed to the acts of violence as compared to their doctor colleagues. Verbal abuse was noted to be the most frequently occurring act of violence and the reasons cited for the same were the unmet needs of patients along with long waiting hours. This study found the relatives to be the most common perpetrators. A Chinese study also found relatives to be common assaulters of the nurses that sometimes the attacks were violent enough to result in the death of nurses [8].

A common scenario in India has been well highlighted by Madhiwalla and Roy in their Mumbai hospital based enquiry report. Following a sudden death in a hospital, the relatives and accompanying people of the patient often lash out at the attending doctors who suffer serious injuries [9]. Similar findings have been reported by other studies which document that aggressive behaviour by the patients or their relatives were followed by feelings of humiliation and helplessness of the doctors [10]. A study in U.K on nurses revealed an upward trend in workplace related harassment [11]. Another U.K based study reported alarming rates of racial and sexual harassment at workplace against the nurses, where more than $70 \%$ reported racial and more than half reported sexual abuse, by their colleagues or patients; in the US 76\% nurses reported being victims of some form of sexual abuse at the work place $[12,13]$. A Europe based study reported an increase in violent events in the hospital against the nurses, citing poor team effort and working shifts related dissatisfaction as major underlying reasons along with insecurities pertaining to the permanence of job $[14,15]$. A 2010 study in Egypt, has found male nurses to be more predisposed to violence at the workplace as compared to the female nurses, verbal abuse being the highest and another observation was an increase in assaults in the night shifts [16].

A study conducted to examine the attitudes of Nurses to workplace violence, in the Obstetrics and Gynaecology departments have observed the relatives of the patient to be the highest contributors of violence (38.5\%) with psychological violence being the most commonly encountered type of violence (78\%) while nurse's factors such as negligence and malpractice were noted to be other factors, around $40 \%$ and $36 \%$ respectively [17]. Studies exploring the incidences of violence against health workers have mostly found nurses to be at an increased risk of incurring violence in comparison to physicians. But few recent studies have found the risk to be almost equal and mostly directed by the visitors of the patients. [18-20]. The familial dynamics are similar in most developing countries and the involvement of family in care giving is quite significant in countries like India. Several similarities can be observed between the Indian and Greek cultures where role of family is concerned. Most Indian and Greek studies have noted the relatives to be the primary perpetrators of violence. Also, factors such as lack of legislative policies and proper protocols to curb instances of violence have been seen to be common determinants of violence against health workers in developing countries, who are at frequent risk in their working environment [21].

\section{To Conclude}

Our study shows that there is a rising trend of violent acts against the nurses at their workplace. Most cases are swept under the carpet and hence the statistics in this regard are far from being accurate. An empathetic approach towards this issue is the need of the hour to safeguard the rights of our Nurses who work tirelessly round the clock. Also, the economic losses incurred by the health sector, the issue of suboptimal health care and adverse psychological impacts can all be reduced if a healthy and fearless working environment is provided to our nursing staff. The first step towards adoption of stringent measures against this violence is the recognition of existing lacunae.

\section{Factors contributing to under reporting}

Co-worker pressure and perceived lack of support

Ignorance regarding the problem (most instances being regarded as not-so-severe)

Cumbersome procedures

Fear of involvement in Medico legal cases

\section{Recommendations for curbing violence in the workplace}

Restrict workplace violence by effective protocols and guidelines.

Improvement of documentation of each and every case reported however seemingly trivial. Strict action against chronic perpetrators (especially co-workers and other hospital staff). Enhancing communication skills of nursing staff and training in stress and anger management. Rehabilitation services for the victims, with job opportunities and financial support. Medical associations and organizations to lend their support and backing to the victim. Investigative services for enforcement of work protocols.

\section{References}

1. Amnesty International: Defense of human rights. http://www.amnesty. org/en/human-rights-defenders/resources/defense-of-human-rights.

2. (2000) American Association of Colleges of Nursing Position statement. Violence as a public health problem. Journal of Professional Nursing 16 : 63-69.

3. Lin Y, Liu H (2005) The impact of workplace violence on nurses in South Taiwan. Int J Nurs Stud 42(7): 773-778.

4. Rippon TJ (2000) Aggression and violence in health care professions. Journal J Adv Nurs 31(2): 452-460.

5. Chapman R, Perry L, Styles I, Combs S (2009) Consequences of workplace violence directed at nurses. Br J Nurs 18(20): 1256-1261.

6. Lyneham J (2000) Violence in NSW emergency departments. Australian Journal of Advanced Nursing 18(2): 8-17.

7. Algwaiz WM, Alghanim SA (2012) Violence exposure among healthcare professionals in Saudi Public Hospitals. A preliminary investigation. Saudi Med J 33(1): 76-82

8. Hongxing YU, Zhenglu Hu, Xifan Zhang, Bin Li, Shangcheng Zhou (2015) How to overcome violence against Healthcare professionals, reduce medical disputes and ensure patient safety. Pak J Med Sci 31(1): 4-8. 
9. Madhiwalla N, Roy N (2006) Assaults on public hospital staff by patients and their relatives: an inquiry Indian Journal of Medical Ethics 3(2): 51.

10. O Brien Pallas L (2009) Creating work environments that are vio-lence free. World Hospitals and Health Services 45: 12-18.

11. Royal College of Nursing (2001) Bullying at work survey, London, UK.

12. NHS Executive (1999) Tackling Racial Harassment in the NHS, Department of Health, London, UK.

13. International Council of Nurses (1999) Guidelines on coping with violence in the workplace, Geneva, Switzerland.

14. Estryn Behar M, Duville N, Menini ML, Camerino D, Le Foll S, et al. (2007) NEXT-Study group. Factors associated with violence against healthcare workers: results of the European Press-NEXT study. Press Med 36(1): 21-35.

15. Martino V (2007) Workplace violence in the health sector: relationship between work stress and workplace violence in the health sector. Ind Health 45(5): 672-678.

16. Moustafa AF, Abbas, Lamiaa A, Fiala, Amira GE, et al. (2010) Epidemiology of Workplace Violence against Nursing Staff in Ismailia Governorate, Egypt. J Egypt Public Health Association 85(1-2): 29-43.
17. Samir N, Mohamed R, Moustafa E, Abou Saif H (2012) Nurses' attitudes and reactions to workplace violence in workplace in obstetrics and gynaecology departments in Cairo hospitals. East Mediterr Health J 18(3): 198-204.

18. Hahn S, Müller M, Needham I, Dassen T, Kok G, et al. (2010) Factors associated with patient and visitor violence experienced by nurses in general hospitals in Switzerland: A cross-sectional survey. J Clin Nurs 19(23-24): 3535-3546.

19. Algwaiz WM, Alghanim SA (2012) Violence exposure among health care professionals in Saudi public hospitals. A preliminary investigation. Saudi Med J 33(1): 76-82.

20. Hegney D, Eley R, Plank A, Buikstra E, Parker V, et al. (2006) Workplace violence in Queensland Australia: The results of a comparative study. International Journal of Nursing Practice 12(4): 220-231.

21. Evmorfia Koukia, Polyxeni Mangoulia, Nikolaos Gonis, Theofanis Katostaras (2013) Violence against health care staff by patient's visitor in general hospital in Greece: Possible causes and economic crisis. Open Journal of Nursing 3(8A): 21-27.
Creative Commons Attribution 4.0 International License

For possible submissions Click Here

\section{Submit Article}

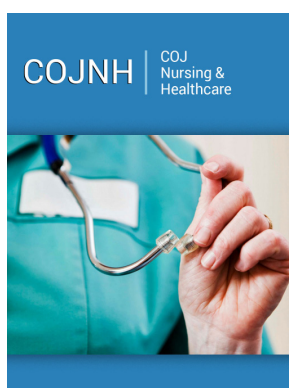

\section{COJ Nursing \& Healthcare}

Benefits of Publishing with us

- High-level peer review and editorial services

- Freely accessible online immediately upon publication

- Authors retain the copyright to their work

- Licensing it under a Creative Commons license

- Visibility through different online platforms 